Children's Hospital, University of Helsinki, Finland. There were 405 patients with an overall male to female ratio of 1.4:1. Patients were grouped according to age: One to 11 months (33), one to four years (139), five to nine years (159), and ten to 16 years (74). The etiology was determined in $69 \%$; it was unknown among one-half of the youngest children and in only one-fourth of those at least ten years of age. The incidence was highest in children younger than two years (16.7/100,000 child years) and was very low in those 14 to 16 years of age $(1 / 100,000$ child years). After the introduction of vaccination against mumps, measles and rubella in 1983, the major encephalitides have been varicella-zoster, Mycoplasma pneumoniae, and respiratory and enteroviruses. Enteroviruses and Herpes simplex were most common in the infants younger than one year of age whereas respiratory viruses and Mycoplasma pneumoniae as well as varicella-zoster dominated in older children. Mumps and varicella were more cormon in boys, and adenovirus and Mycoplasma pneumoniae were more cormon in girls. Seasonal accumulation appeared in February and March and the incidence was lower in July and August. (Koskiniemi M et al. Epidemiology of encephalitis in children: a 20-year survey. Ann Neurol May 1991; $\underline{29}: 492-497$ ).

COMMENT. This study is important in emphasizing a greater interest in childhood encephalitis and the need for early diagnosis and treatment especially in young infants. The Mayo Clinic study in 1984 had shown that encephalitis in young children is often diagnosed late. The authors suggest that convulsions of unknown origin may be due to ill-defined encephalitis caused by respiratory viruses and adenovirus in winter months and enteroviruses in autumn. The etiology is often difficult to establish but antigen tests for respiratory viruses, adenoviruses, Herpes virus, and Mycobacterium pneumoniae are available and will provide the diagnosis within a few hours.

\title{
POSTENCEPHALITIC TOURETTE SYNDROME
}

A six year old girl who developed a Tourette-like syndrame following herpes encephalitis is reported from the Division of Child Neurology, Walter Reed Army Medical Center, Washington, DC and Departments of Neurology and Pediatrics, Johns Hopkins University School of Medicine, Baltimore, MD. A three day prodrame of headaches, malaise, nausea and vomiting was followed by fever and focal motor seizures involving the left side of the face and the left arm. Lumbar puncture showed 122 IBCs ( $80 \%$ lymphocytes) and a protein of $31 \mathrm{mg} / \mathrm{dl}$. A repeat lumbar puncture within 24 hours showed $750 \mathrm{WBCs}, 43 \mathrm{RBCs}$, and protein $85 \mathrm{mg} / \mathrm{dl}$. CT on hospital day three and subsequent MRIs showed a small hemorrhagic lesion in the right mesial temporal region and progressive edematous changes in the right temporal lobe, basal ganglia and thalamus. EEG showed focal slowing over the right temporal and frontal lobes. A presumptive diagnosis of herpes encephalitis was made and Acyclovir was given for ten days. At two weeks after discharge she developed eye blinking and sudden rapid recurrent purposeless nonrhythmic stereotyped motor movements and vocalizations. The tics 
were characterized by facial grimacing, head twitching, and shoulder shrugging plus eye rolling, facial contorsions, jumping, touching objects and body parts and making obscene gestures. Vocal tics included grunting, sniffing, and snorting sounds. The tics were suppressed after several weeks on Pimozide (Orap $1 \mathrm{mg}$ b.i.d.). (Northam RS, Singer HS. Postencephalitic acquired Tourette-like syndrome in a child. Neurology April 1991; $41: 592-593$ ).

COMNENT. The authors refer to reports of acquired Tourette syndrome in adults. Tics followed withdrawal from neuroleptic medications or in association with herpes and other encephalitides, toxic and metabolic encephalopathies, strokes, cerebral tumors, trauma, multiple sclerosis, syphilis, Huntington's, Alzheimer's, and Creutzfeldt-Jakob diseases.

\section{SPINAL CORD SCHISTOSOMIASIS}

A seven year old girl with Schistosoma mansoni infection of the spinal cord mimicking cord neoplasm is reported from the Department of Neurology, University of Michigan, Ann Arbor, MI. Exposure to Schistosomal parasites occurred while bathing in a river in Sierra Leone, Southern Africa. The illness presented with back and leg pain and a low-grade fever. Within 24 hours she had weakness of the leg and four days later she was unable to walk, to urinate or defecate. Sensation was diminished below the low thoracic area. CSF showed 2 RBCs, 250 WBCs with $78 \%$ lymphocytes, protein $584 \mathrm{mg} / \mathrm{dl}$ and glucose $55 \mathrm{mg} / \mathrm{dl}$. Myelography revealed a large intramedullary mass at thoracic levels 11 and 12. MRI with Gadolinium demonstrated enhancement of the cord at T10 level through the conus. Decompressive laminectamy was performed with resection of a yellow gliotic appearing cord of neoplastic appearance. Histologic examination revealed eggs characteristic of $S$. mansoni. After treatment with praziquantel, lower extremity strength gradually improved and six months later, she was walking well with braces. (Selwa LM, Garofalo EA et al. Spinal cord schistosomiasis: A pediatric case mimicking intrinsic cord neoplasm. Neurology May 1991; 41:755-757).

COMMENT. In patients with acute or subacute paraparesis who have been exposed to endemic areas (South Africa, South America, Caribbean or the Middle East), schistosomiasis must be suspected. Spinal cord involvement can be delayed for up to six years after exposure, and findings on MRI and myelography may mimic spinal cord neoplasms. Patients with mild or early involvement of the spinal cord may be benefitted by antiparasitic medications alone and surgery may be unnecessary.

\section{CNS COMPLICATIONS OF RHEUMATOID ARTHRITIS}

Mass lesions in the basal ganglia bilaterally and mainly involving the globus pallidus are reported in a two year old boy with juvenile rheumatoid arthritis from the Department of Pediatrics, Hokushin General Hospital, Nishi, Nagano, Japan. At ten months after the onset of the arthritis he developed a relapse with fever, heart murmur, 